EPJ Web of Conferences 57, 00002 (2013)

DOI: $10.1051 /$ epjconf/20135700002

(C) Owned by the authors, published by EDP Sciences, 2013

\title{
Norman Ramsey and ICAP
}

\author{
D. Kleppner \\ MIT, Cambridge, MA 02139, USA
}

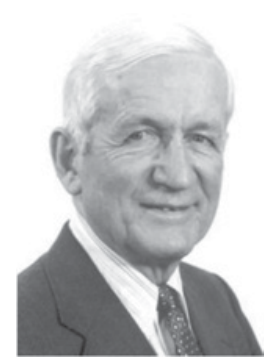

Norman Foster Ramsey, who was born on August 27 th 1917 and died on November $4^{\text {th }}, 2011$, had an enormous influence on the world of physics during the second half of the $20^{\text {th }}$ century. His scientific ideas underlie much of the research reported at this conference and he played a principal role in the creation of ICAP itself. In 1946 Ramsey became the first head of the physics department at the newly created Brookhaven National Laboratory. He appointed Victor A. Cohen, I.I. Rabi's first graduate student, to set up a molecular beams laboratory. Interest in molecular beam magnetic resonance grew rapidly and in the early 1950s, Ramsey, who was then at Harvard, inaugurated a summer conference-the Brookhaven Molecular Beams Conference-that was held biannually and supervised by Victor Cohen. Scientific interest flourished and in the mid 1960s Vernon Hughes proposed expanding the Brookhaven Molecular Beams Conference into the International Conference on Atomic Physics, ICAP. The first ICAP was held at New York University in the spring of 1968. Vernon Hughes is justly remembered as the father of ICAP but Norman Ramsey deserves to be remembered as its grandfather.

Ramsey's name is primarily associated with the separated oscillatory field method for which he received the Nobel Prize in 1989, but this was only one of his many scientific contributions. With Edward M. Purcell he launched the search for breakdowns of fundamental symmetries that continues today and he carried out classic studies of magnetic interactions in molecules. He had a deep interest in high precision measurements and atomic clocks. His invention of the hydrogen maser was cited in his Nobel Prize. That Prize was shared with Hans G. Dehmelt and Wolfgang Paul and at the Nobel banquet Ramsey presented what could be a credo for much of the ICAP community:

Although we are greatly pleased by being recognized individually, we believe the award also symbolically honors our entire field of physics, that of high-precision measurements. The award reminds the world that Physics is an experimental science and can still be successfully pursued in a university laboratory. Many scientists throughout the world are doing beautiful and accurate measurements. We believe that research of this nature will continue to make major contributions to the understanding of the fundamental laws of our universe.

Ramsey graduated from Columbia University in 1935 with a major in mathematics and what he described as "a heavy minor" in physics. He spent two years as an undergraduate at Cambridge University and returned to Columbia at the end of the summer of 1937. He joined I. I. Rabi's group, notwithstanding Rabi's admonition that molecular beams research was scientifically exhausted. A few months later Rabi invented the magnetic resonance method. Rabi's group applied the new method to measure nuclear magnetic moments in the $\mathrm{LiCl}$ and $\mathrm{HD}$ molecules. Unfortunately, the $\mathrm{HD}$ experiments produced what were described as junk spectra. Ramsey, as the youngest student, was handed the task of sorting through the junk while the rest of the group pursued more promising research. Ramsey managed to overcome the experimental problems that were corrupting the HD spectrum and found tantalizing evidence that the deuteron possesses a quadrupole moment. The discovery that the nuclear force is not central generated significant theoretical interest. Initially the spectral resolution was barely adequate to reveal the quadrupole effects but by lengthening the radiofrequency coil used to induce the resonance,

This is an Open Access article distributed under the terms of the Creative Commons Attribution License 2.0, which permits unrestricted use, distribution, and reproduction in any medium, provided the original work is properly cited. 
and hence the interaction time of the molecules in the molecular beam, the resolution became high enough to measure the quadrupole moment precisely.

In 1950, while struggling with the design of a molecular beams apparatus at Harvard, Ramsey hit upon the concept of the separated oscillatory field: applying the radio frequency field by two short coils as far apart as practical, rather than a single long coil. Apparently he initially missed the significance of his invention, for his first reaction was delight that the method produced almost twice the resolution of the previous method whereas it soon opened the way to far greater improvements than that. His method made high-resolution microwave spectroscopy practical because the "coils", really microwave structures, could be short compared to the wavelength while the distance between them could be long. This immediately opened the possibility of creating a hyperfine-based atomic clock and within a few years such a clock-the cesium clock-was demonstrated. Furthermore, the separated oscillatory field method simplified the problem of creating a uniform magnetic field for molecular beam resonance experiments because only the average field, in contrast to the local field, along the line of motion is important. Today, the separated oscillatory field method is usually described in terms of quantum interferometry and the atomic states within the interferometer are recognized as entangled states. Applications of these concepts to metrology, quantum information, and, of course, to atomic clocks, are prominent among the themes of this conference. The talks by Serge Haroche, Edward A. Hinds and Jun Ye, among others, provide compelling evidence for the power of these concepts.

In that same year, 1950, Ramsey and Edward M. Purcell pointed out that there was no experimental evidence that nuclear force were invariant under parity inversion, and later Ramsey pointed out that evidence for time-reversal symmetry was similarly lacking. He started a search for an electric dipole moment in the neutron, and this inspired similar searches for the breakdown of fundamental symmetries. The talk by Ed Hinds describes how the search for an electric dipole moment of the electron has now assumed primary importance in particle physics.

Ramsey's influence in the worlds of physics arose not only from his scientific contributions but also from his roles as a scientific statesman and teacher. Early in his career he worked with I.I. Rabi to establish Brookhaven National Laboratory. Though young, he displayed a remarkable ability to reconcile strong-willed senior colleagues who were deeply committed to locating the new laboratory close to their institutions. He played a similar role in creating the Fermi National Accelerator Laboratory near Chicago in the 1960s. Brookhaven and Berkley were so convinced that each was the only reasonable site for a large new accelerator that the project bordered on collapse. Ramsey brought the opposing sides together and went on to provide the leadership with the U.S. Congress needed to make Fermilab a reality. As the first Science Advisor to NATO, he helped the Les Houches school through a financial crisis, started the programs that supported the Varenna Conferences, and generated fellowships for many young European scientists. As a teacher at Harvard University Ramsey supervised seventy-three Ph. D. students and taught generations of physicists in his classic course on molecular beams. Following his mandatory retirement from Harvard in 1986 he continued to teach as a visiting professor at numerous colleges and universities, and presented freshman seminars at Harvard.

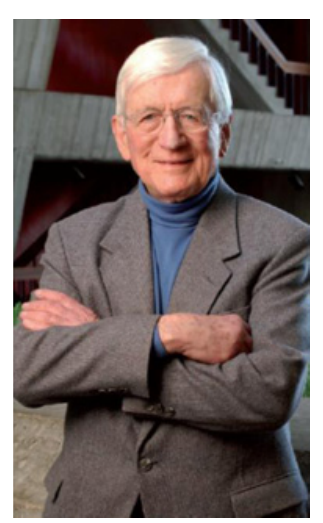

Ramsey left a deep impression on those he met because of the warmth of his personality and his genuine interest in students and colleagues. By his example he taught scientific integrity at the highest level. Not only was he meticulous about scientific clarity and accuracy, he was meticulous about being fair and considerate to his competitors. I believe that many of the instances of generosity and collegiality that are common in our field reflect, either directly or indirectly, the scientific legacy of Norman Ramsey. 